$1-1-2001$

\title{
Beyond pain management: A primer for providing quality end-of- life care
}

\author{
Linda P. Tomko \\ Jefferson Medical College \\ Terri L. Maxwell \\ Jefferson Medical College
}

Follow this and additional works at: https://jdc.jefferson.edu/fmfp

Part of the Family Medicine Commons, and the Translational Medical Research Commons

Let us know how access to this document benefits you

\section{Recommended Citation}

Tomko, Linda P. and Maxwell, Terri L., "Beyond pain management: A primer for providing quality end-of-life care" (2001). Department of Family \& Community Medicine Faculty Papers. Paper 2. https://jdc.jefferson.edu/fmfp/2

This Article is brought to you for free and open access by the Jefferson Digital Commons. The Jefferson Digital Commons is a service of Thomas Jefferson University's Center for Teaching and Learning (CTL). The Commons is a showcase for Jefferson books and journals, peer-reviewed scholarly publications, unique historical collections from the University archives, and teaching tools. The Jefferson Digital Commons allows researchers and interested readers anywhere in the world to learn about and keep up to date with Jefferson scholarship. This article has been accepted for inclusion in Department of Family \& Community Medicine Faculty Papers by an authorized administrator of the Jefferson Digital Commons. For more information, please contact: JeffersonDigitalCommons@jefferson.edu. 


\title{
Beyond Pain Management: A Primer For Providing Quality End-of-life Care
}

\author{
Linda P. Tomko MD \\ Instructor, Assistant Medical Director \\ Department of Family Medicine \\ Jefferson Hospice \\ Jefferson Medical College, Jefferson Health System \\ Terri L. Maxwell RN, MSN, AOCN \\ Executive Director, Center for Palliative Care \\ Department of Family Medicine \\ Jefferson Medical College
}

\section{Citation:}

Linda P. Tomko \& Terri L. Maxwell: Beyond Pain Management: A Primer For Providing Quality End-of-life Care: The Internet Journal of Family Practice. 2001; Volume 1, Number 2.

\section{Abstract}

Providing excellent care for a dying patient is something all patients deserve. Hospices and palliative care centers exist in many areas to aid primary care physicians and patients through this difficult time. It is important to remember that most patients want to prepare for death, if at all possible. Everyone does this in his or her own way, but oftentimes concern about pain and symptom management interfere with this very involved and valuable process. Being prepared to treat these symptoms as well as addressing your patient's emotional needs is imperative. Referral to a hospice, if possible, will only strengthen the support available to the patient, the family, and you, the primary care physician. In the end, there is much that we have to offer a dying patient. Efforts should not stop because the illness cannot be cured. So much can happen to someone in the window of time between terminal diagnosis and death. Making this period one in which a person is as mentally clear, physically comfortable, and symptom free for as long as possible is a goal that is worthy of our efforts. 


\section{Introduction}

"I'm sorry, but there is nothing more that we can do."

Imagine what it must be like to hear those words: so sobering, so isolating, so final, and so untrue. Yet they are spoken and heard far too often in physician offices and hospitals. These words have become a cliché in modern lingo, spoken by movie and TV doctors for dramatic effect. Today, with the advances in palliative care, these words never need to be spoken to a patient. Primary care physicians now realize there is much that can be offered to the patient with advanced disease, and that providing quality and comprehensive care to the dying patient is a skill worth knowing and knowing well.

Accepting death as a natural outcome of illness is antithetical to the way thousands of doctors are trained. Physicians and patients expect physicians to cure and consider death a failure. Pick a physician at any level of training. Chances are that he or she is not only reluctant to speak to a dying patient about death, but is also inaccurate in his/her prognosis. Oftentimes, physicians are overly optimistic about prognoses and patients end up being referred to hospice or palliative care teams much too late. ${ }^{1}$ The SUPPORT Study (Study to Understand Prognosis and Preference for Outcomes and Risks of Treatments) revealed that dying patients also experience considerable suffering and are the victims of inappropriate use of medical resources. ${ }^{2}$ Many people die long and painful deaths and receive unwanted, invasive medical attention. Some people die in a place where the fear of pain and the loss of control and dignity become a burden to them and their families. ${ }^{3}$ It doesn't have to be this way. The ideal environment would be one in which the patient and family would be able to plan ahead for the death and where the center of control is wrested back from the medical sphere and returned to the patient.

Now more than ever, patients have the chance to spend their dying days cared for by a hospice or palliative care team. These teams attempt to provide a secure environment for the patient where symptoms are expertly managed. Such teams provide medical, emotional and spiritual support to the patient as well as his or her entire family through a comprehensive, interdisciplinary approach to care. Hospice/palliative care teams typically include nurses, home health aides, social workers, pastoral care workers, administrators, and physicians. Satisfaction at the end of life has been positively correlated with hospice, where the emphasis is placed on palliation. However, only about $20 \%$ of patients who die in the United States receive hospice care. The admission criterion of a 6-month prognosis or less has been identified as a barrier to hospice referrals. This is in part related to the difficulty in accurately predicting prognosis and in part related to the discomfort surrounding discussing prognosis with patients. ${ }_{-}^{4}$

Becoming a physician capable of providing for the needs of a dying person requires accepting the inevitability of death in some circumstances. Prognosis needs to be objectively and accurately assessed and then shared with the patient and patient's family in a compassionate, clear, and supportive manner. Too often, physicians have inadequate skills to address these conversations. A study by Barzansky, Veloski, Miller, and Jonas 
reviewed the history and status of end-of-life care in US medical schools in 1999 and found that while most medical schools reported that they include end of life care in their curriculum, many medical students describe their education as inadequate. ${ }^{5}$ Medical schools have not included communication skills or palliative care concepts in their curriculum and there is an absence of explicit prognostication in medical textbooks. ${ }_{-}^{6}$ Physicians often fear that discussing a poor prognosis or identifying a condition as terminal will cause emotional pain and loss of hope and when patients suffer emotionally, physicians often do not know how to respond. ${ }^{7}$ Others view death as a failure or feel threatened by such discussions. ${ }^{8}$ Latimer describes ethical communication at this time as consisting of "truth with tenderness." ${ }^{9}$ Information about prognosis should be as accurate as the circumstances allow and conveyed in a language that is understandable to the patient. Euphemisms and medical jargon should be avoided and time should be allowed to answer any questions that the patient may have. The patient should be given referrals to the palliative care team if one is available and desired. Lastly, the physician should be sure that the patient has a way to reach him or her for questions that are thought of later.9

An important aspect of palliative care is establishing the goals of care. Discussions surrounding goals for care should include clear information about treatment choices, including measures to actively support the patient in palliative care. Once a choice has been made, it should be repeated back to the patient for verification. An important care goal is having the patient and family realize their important role in working together with the healthcare team. A pathway for patient support in times of uncertainty should be identified. This includes assisting the patient in writing an advance directive and durable medical power of attorney, as well as helping to iron out complicated family dynamics.

Another important step in becoming a physician who provides comprehensive, quality care for the dying patient is gaining expertise in managing such patients. Recent studies have shown that three out of every four patients dying from cancer experience significant periods of pain. ${ }^{10}$ Appropriately and expeditiously managing pain in the dying patient includes the skillful use of opioids in these circumstances. Many articles have been written on managing pain in the dying patient. Several references are provided at the end of this article.

Providing comprehensive care to the dying patient also means that primary care physicians should be familiar with methods of treating distressing symptoms other than pain. These symptoms; dyspnea, anxiety, depression, gastrointestinal symptoms, anorexia/cachexia, delirium and asthenia are a common to the dying experience and are too often inadequately or poorly managed. The remainder of this article will focus on the medical management of these symptoms.

\section{Dyspnea}

Shortness of breath is a terrifying and common symptom in the dying patient. Dyspnea can frighten patients and family members to the extent that the patient may be sent to the hospital for airway or ventilatory support even if the original plan and goals of treatment 
did not call for such action. Although the rate at which dyspnea occurs has not been well documented, it is believed to occur with moderate severity in one-quarter of all dying patients. ${ }^{11}$ The physician and team caring for the dying individual need to have a plan available for treating dyspnea because panic in the home is often and understandably the rule when someone is acutely short of breath.

Dyspnea can be defined as an uncomfortable awareness of one's breathing. ${ }^{12}$ Dyspnea is now recognized as a combination of many factors including disordered breathing due to a multitude of medical conditions, psychological issues, and functional problems. ${ }^{13}$ Like pain, people with comparable degrees of functional lung impairment may experience varying degrees of dyspnea. Dyspnea is a subjective phenomenon, which is best measured by patient self-report. The objective signs many times do not match the patient's perceptions of dyspnea and other functional limitations. The treatment of dyspnea should address the underlying cause, taking into account the life expectancy, invasiveness, and potential complications of the therapeutic interventions. ${ }^{14}$ Since emotional events, such as anxiety, often play an important role in engendering dypsnea, the goals of treatment are usually two-fold. First, to determine the underlying cause and treat it if possible, and secondly, to treat the patient so that the relief of the symptom is the goal rather than treating a lab or test value. Pulseoximetry is not a reliable indication of dyspnea. The patient's ease of breathing often serves as the best marker. The goal in the treatment of shortness of breath in the dying patient is to improve the subjective symptoms with the treatments available and to keep the patient as comfortable as possible using the patient's own standard for our evaluation.

The standards for treatment of dyspnea have been oxygen, opioids, and benzodiazepines. Oxygen is a great comfort to many people who are short of breath. A number of studies have revealed it to be a great support for the patient and family. ${ }^{15}$ Its benefits for some people go beyond an objective measure such as an increase in $\mathrm{PO} 2$ - it provides them with a level of comfort and a sense of freedom from fear of suffocation. Unless contraindicated by diagnosis, oxygen should be available to the patient who is short of breath.

Opioids have the ability to decrease the physiologic need for oxygen. For many years, palliative care teams in Britain and some in the US have used nebulized opioids, believing that local site treatment was preferable. Recent studies, however, have revealed that nebulized opioids most likely have little effect beyond that of placebo and that nebulized saline itself can be effective for treatment for dyspnea. ${ }^{16}$ When first starting an opioid, it is often preferable to begin with a concentrated, short-acting medication such as Oxyfast or MSIR. 5 to $10 \mathrm{mg}$ of this type of medication in the opioid naive patient is often sufficient to provide relief when combined with oxygen and other therapies for the underlying causative condition.

Benzodiazepines have long played a role in the treatment of dypsnea. Experts in palliative care are split in how and if to prescribe a benzodiazepine for dyspnea. One camp believes that the medication is necessary to treat the anxiety, which inexorably accompanies any attack of dyspnea.1 Others believe that benzodiazepines have limited 
benefits in patients that do not have panic/anxiety periods and are being used too frequently and unnecessarily for shortness of breath.14 Benzodiazepines are best used to treat the anxiety associated with dyspnea in combination with an opioid.

When treating shortness of breath, take into consideration the underlying disease. Patients with airflow obstruction due to chronic obstructive pulmonary disease, for instance, will benefit from treatment with beta-agonists and should be continued on their standing doses of inhalers or nebulized medications.

Patients suffering from end stage lung disease may also have problems handling secretions. Often a dose of levsin or scopalamine can bring this under control, especially if used in conjunction with the appropriate adjunct medications

\section{Anxiety}

Anxiety is common in the dying, as patients face their fears and concerns about their impending death. However, anxiety is not a normal, inevitable consequence of dying and, when present, should be managed aggressively. If anxiety is accepted as a normal part of the dying process, then a person is left to suffer with an extremely painful mental and emotional experience without the aid of medication, personal attention, or therapy. Anxiety may also mask incipient medical complications such as pain, internal injuries, or pulmonary embolism. Risk factors for anxiety include organic mental disorders, concurrent life events or social difficulties, lack of support and understanding from one's family and friends, and apprehension and worry. $\underline{17}$

The treatment of anxiety often requires a combination of psychosocial support and pharmacotherapy. Providing support and a safe environment for discussing one's concerns and fears is very important. Sometimes a simple question, "What are you worried about?" allows a patient to discuss his or her concerns, which may be therapeutic unto itself.

If pharmacotherapy is required, benzodiazepines are generally the medication of choice. The benzodiazepines are chosen based upon the desired half-life. The longer half-life medications have a more sustained effect, but may accumulate and are not recommended in the elderly or those with hepatic impairment. Any of these agents needs to be used with caution, since delirium can be readily induced by a combination of drugs and illness in dying patients. Short-acting benzodiazepans (lorazepam, oxazepam) are appropriate to use first line, but if they are not working or cannot be used, major tranquilizers such as haloperidol may be helpful. When initiating haloperidol, always begin with small doses: 1.5 to $5 \mathrm{mg}$ every six to eight hours as needed. Patients should be closely monitored when taking such medication. If anxiety persists, adjunctive therapies such as acupuncture and guided imagery with relaxation therapy may also help. In cases of intractable anxiety, a psychiatric consultation utilizing new drugs and therapies may prove beneficial. 


\section{Depression}

Although sadness is common in patients with life-threatening disease, it is a myth that feeling helpless, hopeless, and depressed are inevitable consequences of advanced illness. Sadness generally responds to supportive interventions. Depression, on the other hand, is a pathologic state marked by greater cognitive impairment and generally requires more aggressive therapy.

Diagnosis can be difficult, but several studies have revealed that merely asking the patient "Are you depressed?" can actually identify people who otherwise might not have received therapy. ${ }^{18}$ Depression should be considered if a person has persistent periods of intense sadness and anxiety accompanied by depressive symptoms for greater than a few days or weeks.

It is well known that the most frequently used drugs for depression, the SSRIs, take several weeks to develop a therapeutic effect. Occasionally, someone does not have weeks to wait for a significant improvement. More and more physicians are discovering the benefits of a class of drugs called psychostimulants (dexamphetamine and methlyphenidate). They are safe and effective and their rate of onset of action is very fast. ${ }_{19}^{19}$ Oftentimes, these medications, when used at low dose and with close supervision, can greatly improve the quality of a patient's last few months. The improvement in energy, concentration, and mood may last until the longer acting drugs are able to take effect.

One should not discount the importance of therapy and counseling in the treatment of the depressed, dying patient. While group therapy may sometimes be helpful, almost any intervention may help the patient to feel better and to be better prepared to cope with daily stressors.

\section{Gastrointestinal Complaints}

Nausea can be a frequent and unwanted companion of a dying person. While frequently related to medication such as chemotherapy and opioids, it is important to attempt to discover the etiology of the nausea to see if specific, targeted therapies can improve symptoms. The most common causes of nausea include autonomic dysfunction, gastroparesis, and opioid analgesics. Opioids cause nausea in several ways, including delayed gastric emptying time, vestibular stimulation, and constipation. ${ }^{20}$ Treatment of nausea depends, in a large part, upon the presumed underlying cause. When the mechanism or cause of the nausea is understood, the treatment can be tailored with improved success in controlling the symptom. There are many drugs used to treat nausea, but for patients without bowel obstruction, metoclopramide is beneficial because it acts centrally and aids in gastric emptying. ${ }_{-}^{21}$ When obstruction is present and increased intestinal and gastric secretions accumulate, medications such as octreotide will help treat the accompanying nausea. ${ }^{22}$ Haloperidol and prochlorperazine act centrally to decrease 
nausea and are frequently also used. A combination of approaches is necessary in some patients.

\section{Delirium}

Perhaps one of the most difficult symptoms to manage in the dying patient is delirium. It is often as difficult to diagnose as it is to treat, but it remains the most frequent neuropsychiatric disorder that affects the dying. Indeed, according to many studies, most people are in a delirious state when they die. ${ }^{23}$ Delirium is a state of decreased cognitive abilities. Delirium usually has a quick onset and in contrast to dementia, is considered to be a potentially reversible process. Patients often exhibit changes in their sleep and wake cycles and may experience a fluctuating level of consciousness. Delirium can be divided into two categories: hypoactive and hyperactive. In the hypoactive phase patients may become more withdrawn, experience difficulty in attending to tasks, or have acute problems with their memory. In the "hyperactive" phase of delirium, patients may experience psychosis with delusions, paranoia, and hallucinations. The differential diagnosis for delirium is extensive. It includes adverse effects of medications (narcotics, benzodiazepines, coriticosteroids and anti-cholinergic agents), infection, metabolic problems, dehydration, direct effects of the disease on the central nervous system, and organ failure. Patients with delirium have a much higher morbidity rate than those without delirium. $\stackrel{24}{\underline{4}}$

In any patient showing acute onset of agitation, impaired cognitive function, altered attention span, or fluctuating level of consciousness, a diagnosis of delirium should be considered. A mini-mental exam is helpful to document the change in mental capacity, and a search for the etiology of the delirium should be undertaken. If a diagnosis for the change is found (i.e. a UTI), this should be treated if desired so by the patient and/or the patients' s family. Approximately $30 \%$ of people with delirium will experience improvement back to baseline with treatment of the immediate cause. ${ }^{25}$ Unfortunately, in the majority of those affected, an etiology will not be found and treatment will not improve their outcome.

Since the cause of delirium is often multifactorial or elusive, and the work-up may be limited by the setting or uncomfortable for the patient, the focus of the care is often patient comfort. If someone is agitated, "sundowning," or delusional, the first line of therapy will involve using neuroleptics. Neuroleptics, such as low-dose haldol, allow the person to think more clearly and to become less agitated with some mild sedation. It is important to remember when using any drug; you want to begin with the smallest dose possible for the situation and the patient. Often $0.5 \mathrm{mg}$ to $1 \mathrm{mg}$ of haloperidol is sufficient to relieve the mental anguish, but this may need to be increased. Other medications that can be used include respiridone, which has an improved side effect profile, but is still investigational in this population.

"Terminal anguish,"12 is a combination of delirium, anxiety, and debilitation that can occur shortly before death. It can be extremely frightening for the patient and extremely 
disturbing to the family. Keeping the patient comfortable, calm, and sedated if necessary should be the major goal of treatment. Pain medication and anxiolytics should be used. Preparatory discussions with family members may be one way to make this time less difficult for everyone involved.

\section{Cachexia/Anorexia}

Perhaps one of the most physically remarkable aspects of the dying process is the weight loss and related change in appearance that many people experience. This cachetic appearance is often alarming and is again usually multifactorial in cause. Anorexia and cachexia seem to travel hand in hand. The physical processes that lead to weight loss include lipolysis, loss of viseral and skeletal protein mass, and profound anorexia. $\underline{26}$ Weight loss decreases a person's chance of survival and has a terrible effect on the morale and psyche of the patient and their loved ones. Aggressive efforts to feed a dying patient (tube feeding, TPN) have not shown significant improvement or extension of patient survival. $\underline{27}$

The treatment of anorexia should be patient driven. If a person requires encouragement for feeding and is still capable of eating, they should be offered food that they enjoy and find appetizing. Pharmacologic measures for therapy are slowly expanding and include corticosteroid and progestational drugs such as megestrol. Corticosteroids have been found to have a limited efficacy for management of this symptom, although they can help alleve asthenia. The optimal doses for Megace and the like are relatively high: between 480 to $800 \mathrm{mg}$. In the dying patient, doses in the $480 \mathrm{mg}$ range may be helpful in stimulating appetite without associated weight gain. Studies performed on these drugs in dying patients report improved appetite, less fatigue, and greater sense of well being. $\underline{28}$ The physician and patient should treat these drugs with the respect that they deserve however, and one must be cautious when using them. The progestation agents can be first line, but it is important to know that if they are stopped too quickly, peripheral edema, thromboembolic events and vaginal bleeding, while rare, are potential side effects. Agents under investigation for the treatment of anorexia include thalidomide and omega 3 fatty acids.

\section{Asthenia}

Another extremely common symptom in the dying patient is asthenia. Although especially prevalent in cancer patients, asthenia can plague almost any person as they approach the end of their life. Brea describes asthenia as a profound tiredness after one performs usual or minimal effort. It is also characterized by generalized weakness. It is estimated that full nine out of ten advanced cancer patients will experience asthenia as their disease progresses. The cause is felt to be multifactorial and is well correlated with anorexia. Tumor effects such as tumor mediated products, anemia, chronic infection and paraneoplastic syndromes often play a role in the development of asthenia.7 Interestingly, 
a study in the Netherlands, where euthanasia is permitted, described asthenia, or "weariness with life" as a frequent contributor to the decision to end one's life. $\underline{29}$

Treatment is based initially upon determining if any specific causes exist. Depression should be treated if present. Infection and anemia should be treated, if the patient desires. If these measures are unsuccessful, non-pharmacologic management should be initiated. Consultations for energy conservation techniques with physical and occupational therapists should be explored and activities of daily living should be modified. It further improvement is still needed, pharmacologic measures should be considered including corticosteroids such as decadron or medrol or methamphetamines such as ritaline. $\underline{30}$ Often, these medications have a limited period of effectiveness in a particular patient, but the quality of time afforded the patient is well worth the trial.

\section{The end of life...how do you know when it's happening}

Families and patients often search for someone to give them absolutes. When will he die? Will it be quick? Will it be painful? While we can attempt to control the symptoms, we cannot always predict the pattern. While it is probably not advisable to give definitive prognoses (e.g. "She'll die before year's end") you can sometimes help patients and their families by informing them when death appears imminent. Certainly, many people die without giving us these clues to pass on to the family, but when they are present, the family should be notified. Signs that the end may be approaching include: terminal breathing patterns such as Cheyne-Stokes respirations, decreased or non-existent urine output, inability to take in food and liquid in a person who has no feeding tube, and declining cognitive status. Talking to family members, letting them know how the pattern has changed, and giving them the opportunity to be with their loved one and say goodbye or "I love you" is an important role that is often overlooked, especially if the patient is not enrolled in a palliative care or hospice program.

\section{Conclusions}

Providing excellent care for a dying patient is something all patients deserve. Hospices and palliative care centers exist in many areas to aid primary care physicians and patients through this difficult time. It is important to remember that most patients want to prepare for death, if at all possible. Everyone does this in his or her own way, but oftentimes concern about pain and symptom management interfere with this very involved and valuable process. Being prepared to treat these symptoms as well as addressing your patient's emotional needs is imperative. Referral to a hospice, if possible, will only strengthen the support available to the patient, the family, and you, the primary care physician. 
In the end, there is much that we have to offer a dying patient. Efforts should not stop because the illness cannot be cured. So much can happen to someone in the window of time between terminal diagnosis and death. Making this period one in which a person is as mentally clear, physically comfortable, and symptom free for as long as possible is a goal that is worthy of our efforts.

\section{References}

1. Billings, JA. Palliative Care. BMJ 2000; 321:555-558.

2. The SUPPORT Principal Investigators. A controlled trial to improve care of seriously ill hospitalized patients: the study to understand prognoses and preferences for outcomes and risks of treatments (SUPPORT). JAMA 1995;274:1591-1598.

3. Council on Scientific Affairs. Good Care of the Dying. JAMA 1996; 275(6): 474-478.

4. Frantz T, Lawrence J, Somov P, Somova M. Factors in hospice patients' length of stay. Am J Hospice and Pall Care, 1999; 16:449-454.

5. Barzansky B, Veloski J, Miller R, Jonas H. Education in end of life care during medical school and residency training. Academic Medicine, 1999;74(10S):S102-S104.

6. Quill T. Initiating end-of-life discussions: addressing the "elephant in the room." JAMA, 2000; 284: 2502-2507

7. Steinhauser K, Christakis N, Clipp E, McNeilly M, McIntyreL, Tulsky J. Factors considered important at the end of life by patients, family physicians, and other care providers. JAMA 2000; 284:2476-2482.

8. Larson DG, Tobin DR. End-of-life conversations: evolving practice and theory. JAMA, 2000; 284:1573-1577.

9. Latimer, EJ. Ethical care at the end of life. Can Med Assoc J. 1998; 158:1741-1747.

10. Coyle N, Adelhardt J, Foley KM, Portenoy RK. Character of terminal illness in the advanced cancer patient: pain and other symptoms during the last four weeks of life. $\mathrm{J}$ Pain Symptom Manage, 1990; 5:83-93.

11. Reuben DB, Mor V. Dyspnea in terminally ill cancer patients. Chest, 1986; 89:234236.

12. Bruera E, Neumann CM. Management of specific symptom complexes in patients receiving palliative care. Can Med Assoc J, 1998;158:1717-1726. 
13. Bredin M, Corner J, Krishnasamy M, Plant H, Bailey C, A'Hern r. Multicentre randomized controlled trial of nursing intervention for breathlessness in patients with lung cancer. BMJ, 1999; 318:901-904.

14. Bruera E, Ripanmonti C. Dyspnea in patients with advanced cancer. In: Berger AM, Portenoy RK, Weissman DE, editors. Principles and practice of supportive oncology. Philadelphia: Lippincott-Raven Publisher; 1998. P. 295-308.

15. Bruera E, Schoeller T, MacEachern T. Symptomatic benefit of supplemental oxygen in hypoxemic patients with terminal cancer: the use of the $\mathrm{N}$ of 1 randomized controlled trial. J Pain Symptom Manage, 1992;7:365-368.

16. Leung R, Hill P, Burdon J. Effect of inhaled morphine on the development of breathlessness during exercise in patients with chronic lung disease. Thorax 1996;51:596600 .

17. Barraclogh J. ABC of palliative care: Depression, anxiety, and confusion. BMJ 1997;315:1365-1368.

18. Chochinov HM, Wilson KG, Enns M, Lander S. Are you depressed? Screening for depression in the terminally ill. Am J Psychiatry, 1997; 154:674-676.

19. Block SD. Assessing and managing depression in the terminally ill patient. Ann Intern Med, 2000;132:209-218.

20. Bruera E, Neumann, C. Management of specific symptom complexes in patients receiving palliative care. Can Med Assoc J, 1998;158:1717-1726.

21. Bruera, E, Seifert L, Watanabe S, Babul N, Darke A, Harsanyi Z, et al. Chronic nausea in advanced cancer patients: a retrospective assessment of a metoclopramidebased antiemetic regimen. J PainSymptom Manage, 1996;11:147-153.

22. Mercadante S. The role of octreotide in palliative care. J Pain Symptom Manage, 1993;9:406-411.

23. Breitbart E, Bruera E, Chochinov H, Lynch M. A National Cancer Institute of Canada Workshop on symptom control, and supportive care in patients with advanced cancer: methodological and administrative issues. Neuropyschiatric syndromes and psychological symptoms in patients with advanced cancer. J Pain Symptom Manage, 1995: 10(2.): 131141.

24. Mazzocato C, Stiefel F, Buclin T. Psychopharmacology in supportive care of cancer: a review for the clinician. Support Care Cancer, 1999; 89-97.

25. Pereira J, Hanson J, Bruera E. The frequency and clinical course of cognitive impairment in patients with terminal cancer. Cancer, 1997;70:835-842. 
26. Bruera E. ABC of palliative care: anorexia, cachexia, and nutrition. BMJ, 1997;315:1219-1222.

27. Klein S, Koretz RL. Nutrition support in-patients with cancer: What do the data really show? Nut Cline Precut, 1994; 9:91-100.

28. Gagnon B, Bruera E. A review of the drug treatment of cachexia associated with cancer. Drugs, 1998;55:675-678.

29. Van der Maas PJ, Van Delden JJM, Pijnenborg L, Looman CWN. Euthanasia and other medical decisions concerning the end of life. Lancet, 1991;338:669-674.

30. Wantanabe S, Bruera E. Anorexia and cachexia, asthenia and lethary. Hematol Oncol Clin North Am, 1996;10:189-206. 